\title{
Eosinophilic pneumonia associated with pirfenidone therapy
}

To the Editor:

Pulmonary eosinophilia is a common manifestation of drug-induced lung disease and, at the time of writing, some 170 medications have been implicated in eosinophilic pneumonia [1]. Since the reaction occurs rarely for any given drug, however, establishing a causal relationship is difficult, and relies on reports of temporal relationship and exclusion of alternative causes of eosinophilic lung disease. Pirfenidone is an oral antifibrotic drug that is conditionally recommended as therapy for patients with idiopathic pulmonary fibrosis (IPF), based on the results of three randomised controlled trials [2]. Here, we describe what we believe to be the first report of the association of pirfenidone therapy with eosinophilic pneumonia.

The patient was a 78-year-old Caucasian man who provided written informed consent to have his case history presented. He had a history of IPF diagnosed 3 years previously, diagnosed according to consensus guidelines [2], on the basis of a high-resolution chest computed tomography (CT) pattern indicative of usual interstitial pneumonia (UIP), and negative history and serological studies for other causes of diffuse parenchymal lung disease. At baseline, he could walk $350 \mathrm{~m}$ in $6 \mathrm{~min}$ with oxygen saturations remaining above $93 \%$ and, 3 weeks before initiation of pirfenidone, his pulmonary function tests (PFTs) showed forced vital capacity (FVC) $3.28 \mathrm{~L}$ (77\% of predicted), forced expiratory volume in $1 \mathrm{~s}$ (FEV1) $2.40 \mathrm{~L}$ (78\% of predicted) and diffusing capacity of the lung for carbon monoxide (DLCO) $10.98 \mathrm{~mL} \cdot \mathrm{min}^{-1} \cdot \mathrm{mmHg}^{-1}(39 \%$ of predicted). 5 weeks after initiation of pirfenidone, he developed progressively worsening dyspnoea on exertion and presented to the clinic 2 weeks later. He had not had fever or sputum production. His other medications were simvastatin and pantoprazole. He did not smoke, had had no recent respiratory exposures, and did not use over-the-counter medications, recreational drugs or alternative therapies. He had no history of asthma, history of travel, or exposures to suggest parasitic diseases. His physical examination revealed only the previously noted bibasilar coarse crackles. PFTs showed a marked decline in FVC and gas transfer (FVC $2.47 \mathrm{~L}$ (58\% of predicted), FEV1 $1.86 \mathrm{~L}$ ( $60 \%$ of predicted) and DLCO $7.01 \mathrm{~mL} \cdot \mathrm{min}^{-1} \cdot \mathrm{mmHg}^{-1}(25 \%$ of predicted)). During a hall walk study, he had a new requirement for supplemental oxygen on exertion: he had oxygen desaturation to $88 \%$ after walking $100 \mathrm{~m}$ on room air, and required $6 \mathrm{~L} \cdot \mathrm{min}^{-1}$ supplemental oxygen to walk $300 \mathrm{~m}$ and maintain saturations above $90 \%$. Laboratory studies showed a normal white blood cell count without eosinophilia, and normal serum chemistries, liver panel and brain natriuretic peptide, but an elevated D-dimer concentration of $443 \mathrm{ng} \cdot \mathrm{mL}^{-1}$ (normal $<231 \mathrm{ng} \cdot \mathrm{mL}^{-1}$ ). A CT pulmonary angiogram revealed no evidence of pulmonary embolism but new multifocal areas of ground-glass opacification in the lower lobes, superimposed on the previously noted UIP pattern (figure $1 \mathrm{a}$ and b). Bronchoscopy and bronchoalveolar lavage (BAL) revealed a cell count of $2.7 \times 10^{5}$ cells per $\mathrm{mL}$ with differential counts of $22 \%$ eosinophils, $11 \%$ lymphocytes, $2 \%$ neutrophils and $62 \%$ macrophages. Bacterial, fungal, viral and mycobacterial cultures, PCR for respiratory viruses, and direct fluorescent assay for Pneumocystis jiroveci

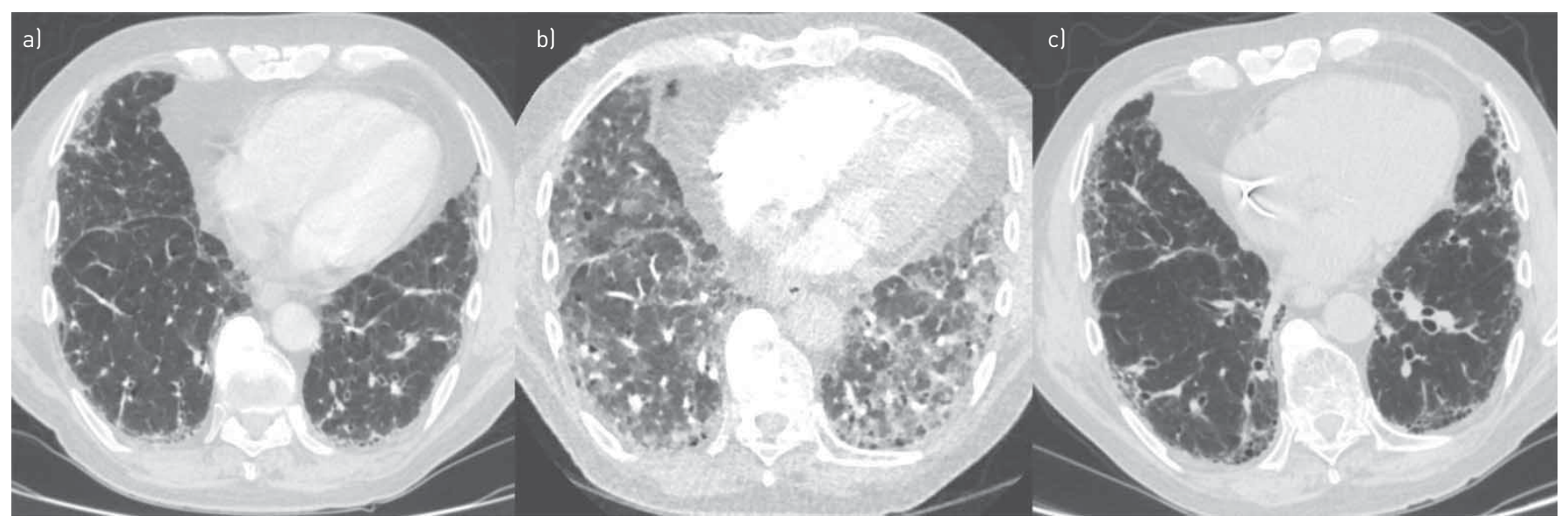

FIGURE 1 Representative axial chest computed tomography (CT) images. a) 2 years before initiation of pirfenidone, a high-resolution study showed peripheral reticulation and honeycombing. b) 7 weeks after initiation of pirfenidone, CT pulmonary angiogram showed superimposed multifocal ground-glass opacities. c) 8 weeks after discontinuation of pirfenidone and initiation of corticosteroids, a high-resolution study showed resolution of ground-glass opacities. 
were negative. Pirfenidone was discontinued and, given the abrupt deterioration with evidence of eosinophilic pneumonia, prednisone started at a dose of $40 \mathrm{mg}$ a day. 8 weeks later, his dyspnoea had resolved and PFTs had improved significantly (FVC $2.99 \mathrm{~L}$ (70\% of predicted), FEV1 $2.35 \mathrm{~L}$ (76\% of predicted) and DLCO $10.86 \mathrm{~mL} \cdot \mathrm{min}^{-1} \cdot \mathrm{mmHg}^{-1}$ (38\% of predicted)), and chest CT showed resolution of ground-glass opacities (figure 1c). Prednisone was tapered off and, 9 months later, he remained well.

Exacerbations of IPF are defined as unexplained respiratory deteriorations of $<30$ days duration associated with new bilateral ground-glass opacities or consolidation superimposed on prior reticular opacities, but the diagnosis requires the exclusion of other identifiable causes of lung injury, which most commonly include infections, cardiogenic pulmonary oedema and pulmonary thromboembolism [3, 4]. The histology of IPF exacerbation shows diffuse alveolar damage superimposed on UIP and, importantly, the condition is not associated with eosinophilia, with BAL fluid containing $<5 \%$ eosinophils reported in the literature, similar to patients with IPF without exacerbation [4,5]. Drug-induced lung toxicity, however, is a common cause of pulmonary eosinophilia, typically together with elevated BAL lymphocytes [6], and is a potentially important diagnostic consideration in evaluating possible IPF exacerbations. The suggested diagnostic criteria for drug-induced eosinophilic lung disease are: 1) absence of other likely causes of lung disease; 2) compatible symptoms; 3) time-course; 4) consistent BAL or histological findings; and 5) improvement after drug discontinuation, with all five criteria indicating a definite diagnosis and four criteria considered a probable diagnosis [7]. Our patient met all of these criteria.

In considering other causes of deterioration in this case, we noted that, since its commercial release in 1992, simvastatin has been associated with eosinophilic pneumonia in a single case report [8]. In our patient, therapy with simvastatin began 5 years prior to his acute illness and was continued for 9 months after discontinuation of pirfenidone without recurrence of eosinophilic pneumonia. We therefore considered simvastatin an unlikely culprit in his eosinophilic lung disease.

There have been two prior reports of respiratory side-effects of pirfenidone to date. A post-marketing surveillance report by the manufacturer describes cases of facial angio-oedema and wheezing associated with the drug, but the number of cases was not reported [9]. A recent report described two patients who developed worsening respiratory symptoms and decline in PFTs shortly after initiation of pirfenidone, with resolution of symptoms after cessation of the drug; one patient had wheezing and cough, and the second developed new ground-glass opacities. BAL cell counts were not reported in these cases [10]. It is possible that all of these cases, including the present report, represent a T-helper cell type 2 allergic response to the drug but this hypothesis awaits further investigation.

In conclusion, pirfenidone therapy may be associated with eosinophilic lung disease. Importantly, this association may be underdiagnosed and underreported since it is indistinguishable from IPF exacerbation. More broadly, eosinophilic pneumonia and drug-induced lung diseases are important considerations in the differential diagnosis of IPF exacerbations, and this report emphasises the importance of a thorough workup, including BAL, for respiratory deteriorations in interstitial lung diseases.

@ERSpublications

Pirfenidone therapy may cause eosinophilic lung disease http://ow.ly/xWyh301icn8

Diana C. Gomez ${ }^{1}$ and Borna Mehrad ${ }^{1,2}$

${ }^{1}$ Interstitial Lung Disease Program, Division of Pulmonary and Critical Care Medicine, University of Virginia, Charlottesville, VA, USA. ${ }^{2}$ The Carter Center for Immunology, University of Virginia, Charlottesville, VA, USA.

Correspondence: Borna Mehrad, Interstitial Lung Disease Program, Division of Pulmonary and Critical Care Medicine, University of Virginia, PO Box 800546, Charlottesville, VA 22908, USA. E-mail: mehrad@virginia.edu

Received: April 232016 | Accepted after revision: May 302016 | First published online: July 072016

Support statement: This study was supported by US National Institutes of Health grants HL098526 and HL098329. Funding information for this article has been deposited with the Open Funder Registry.

Conflict of interest: None declared.

\section{References}

1 Camus P. Pneumotox: the Drug-Induced Respiratory Disease Website. Version 488. www.pneumotox.com Date last updated: April 23, 2016. Date last accessed: April 23, 2016.

2 Raghu G, Rochwerg B, Zhang Y, et al. An official ATS/ERS/JRS/ALAT clinical practice guideline: treatment of idiopathic pulmonary fibrosis. An Update of the 2011 clinical practice guideline. Am J Respir Crit Care Med 2015; 192: e3-e19.

3 Collard HR, Moore BB, Flaherty KR, et al. Acute exacerbations of idiopathic pulmonary fibrosis. Am J Respir Crit Care Med 2007; 176: 636-643. 
4 Raghu G, Collard HR, Egan JJ, et al. An official ATS/ERS/JRS/ALAT statement: idiopathic pulmonary fibrosis: evidence-based guidelines for diagnosis and management. Am J Respir Crit Care Med 2011; 183: 788-824.

5 Lee JS, Song JW, Wolters PJ, et al. Bronchoalveolar lavage pepsin in acute exacerbation of idiopathic pulmonary fibrosis. Eur Respir J 2012; 39: 352-358.

6 Allen JN. Drug-induced eosinophilic lung disease. Clin Chest Med 2004; 25: 77-88.

7 Solomon J, Schwarz M. Drug-, toxin-, and radiation therapy-induced eosinophilic pneumonia. Semin Respir Crit Care Med 2006; 27: 192-197.

8 Yoshioka S, Mukae H, Ishii H, et al. [A case of drug-induced pneumonia possibly associated with simvastatin]. Nihon Kokyuki Gakkai Zasshi 2005; 43: 600-604.

9 European Medicines Agency. Esbriet: EPAR Product Information. www.ema.europa.eu/docs/en_GB/document_ library/EPAR_-_Product_Information/human/002154/WC500103049.pdf Date last updated: 2015. Date last accessed: September 11, 2015.

10 Campainha S, Nogueira C, Costa F, et al. Not yet known side effects of pirfenidone in the treatment of idiopathic pulmonary fibrosis? Rev Port Pneumol 2016; 22: 126-127.

\title{
Can intermediate monocytes predict response to infliximab therapy in sarcoidosis?
}

\author{
To the Editor:
}

Sarcoidosis is a systemic granulomatous disease of unknown origin that can cause a variety of symptoms, but most often affects the lungs [1]. Because sarcoidosis is self-limiting in the majority of patients, not all patients require therapy [2]. In case of severe sarcoidosis, first-line therapy consists of prednisone, with methotrexate or azathioprine being the most commonly used second-line options [3]. Infliximab, a monoclonal anti-tumour necrosis factor (TNF) drug, is an effective third-line therapeutic for severe sarcoidosis $[4,5]$.

The exact nature and order of immunological events leading to the formation of sarcoid granulomas remains unknown. Granulomas consist of highly differentiated mononuclear phagocytes (mainly activated macrophages) and lymphocytes [1]. Macrophages of sarcoidosis patients are known to produce excessive amounts of TNF compared with healthy controls $[6,7]$. The importance of TNF is underlined by the success of infliximab therapy. As monocytes are the precursors of tissue macrophages, monocytes might be a key player in this process. A promising new therapeutic strategy for sarcoidosis is the combined monocyte and granulocyte apheresis [8]. Although only seven patients were included in a pilot study, the positive effects underline the importance of monocytes in the sarcoidosis disease process.

CD16-expressing monocytes are expanded in various immune-mediated diseases, such as rheumatoid arthritis, diabetes, atherosclerosis, bacterial infections and HIV [9]. High percentages of CD16-expressing monocytes were also found in sarcoidosis patients when compared with controls $[10,11]$. CD16-expressing monocytes, and intermediate monocytes in particular, fulfil a pro-inflammatory role by producing TNF as well as other cytokines [12]. We have previously shown that sarcoidosis patients have a significantly higher percentage of the pro-inflammatory intermediate monocytes than healthy controls [13], an observation that has been confirmed recently by Hofer et al. [14]. To date, the few available studies investigating monocytes in sarcoidosis have focused solely on monocyte subsets while any potential relationship between disease activity or response to therapy has not yet been addressed.

In our present study, we analysed whether monocyte subsets in blood can predict the clinical response of sarcoidosis patients to therapy with infliximab (Remicade; Centocor Inc., Malvem, PA, USA). To this end we have studied a selected group of patients with severe sarcoidosis, who were unresponsive to first- and second-line treatment or experienced severe side-effects. These patients received infliximab intravenously according to a standardised protocol starting with $5 \mathrm{mg} \cdot \mathrm{kg}^{-1}$ at weeks 0 and 2 and then every 4 weeks for a period of 26 weeks. This study is part of a prospective, open-label cohort study by VorselaARs et al. [15] evaluating the efficacy of infliximab in sarcoidosis. The institutional review board and the ethics committee approved the study and all patients gave written informed consent.

Peripheral mononuclear blood cells from 38 sarcoidosis patients and 18 healthy controls were stained for CD14, CD16 (eBioscience, San Diego, CA, USA) and HLA-DR (human leukocyte antigen-antigen D related) 